Article

\title{
Incorporating Children and Young People's Voices in Child and Adolescent Mental Health Services Using The Family Model
}

\author{
Benjamin Hoadley ${ }^{1, *}$, Freya Smith ${ }^{1}$, Cecilia Wan ${ }^{2}$ and Adrian Falkov ${ }^{1}$ \\ ${ }^{1}$ Child \& Youth Mental Health Service, Royal North Shore Hospital, Sydney, NSW 2065, Australia; E-Mails: \\ benjamin.hoadley@health.nsw.gov.au (B.H.), freya.smith@health.nsw.gov.au (F.S.), adrian.falkov@health.nsw.gov.au (A.F.) \\ 2 The Brolga Unit, Hornsby Hospital, Sydney, NSW 2077, Australia; E-Mail: cyswan@med.usyd.edu.au \\ * Corresponding author
}

Submitted: 28 March 2017 | Accepted: 17 July 2017 | Published: 26 September 2017

\begin{abstract}
Mental illness in children and young people is increasing in frequency and complexity, is emerging earlier and is persisting into adulthood. This is a global issue with implications for research, policy and practice. Children and young people require the experience of safe, nurturing relationships for optimal lifelong outcomes. Despite awareness of this in Child and Adolescent Mental Health services, a focus on the relational context in which children and young people present is not universal. A challenge in family focused practice is to ensure that no individual's voice is 'too loud' and that children and young people's voices are heard. This article illustrates how a balance between individual and systems understanding can be achieved in therapeutic work by incorporating the voices of children and young people and concerns of other family members. This article describes an approach to improving family focused practice in a public Child and Adolescent Mental Health service. Use of The Family Model, as a family focused practice tool, is presented across three service settings. The Family Model intervention is briefly described, outlining the way in which it supports collaborative practice and assists clinicians to achieve the balance described above. Vignettes will demonstrate how children and young people's voices are explicitly incorporated in formulating mental health issues with two generations to generate developmentally informed care plans.
\end{abstract}

\section{Keywords}

adolescent; children; family focused practice; mental health; psychology; The Family Model; young people

Issue

This article is part of the issue "Promoting Children's Participation in Research, Policy and Practice", edited by Jo Aldridge (Loughborough University, UK).

(C) 2017 by the authors; licensee Cogitatio (Lisbon, Portugal). This article is licensed under a Creative Commons Attribution 4.0 International License (CC BY).

\section{Introduction}

Incorporating children and young people's (Ch\&YPs') voices in mental health (MH) services is necessary and challenging. In Child and Adolescent Mental Health (CAMH) services, it is assumed that Ch\&YPs' views are routinely incorporated into usual care. Indeed, as the British Medical Association points out, developmentally appropriate shared decision-making has been a goal of such services (as cited in Rutter \& Stevenson, 2010, p. 9) and this right of young people to have their perspective considered and taken into account in decision-making is protected in article 12 of the United Nations Convention on the Rights of the Child $(1989$, p. 4) and is reflected in local policy (Australian Infant, Child, Adolescent and Family Mental Health Association, 2008, p. 15) and legislation, which emphasises that Ch\&YPs' views are to be considered with respect to their developmental capacity (Children and Young Persons (Care and Protection) Act No 157,1998, p. 6). The attention given to consumer participation in CAMH research was described as limited a decade ago (Macdonald et al., 2007, p. 3) and though the importance of Ch\&YP participation featured in health policy at the time, knowledge concerning the pragmatics 
of Ch\&YP involvement was also in its infancy (Day, 2008, p. 6). The literature in this field is evolving and though Ch\&YP participation is not routine, it is now recognised that Ch\&YP attain value and benefit from such participation (Weil, Lemer, Webb, \& Hargreaves, 2015, p. 1).

A related priority of CAMH services is the provision of family focused care, as demonstrated by government and service provider investment and health policy (Foster et al., 2016, p. 129). This in part reflects the recognition of family functioning and the interplay between presenting symptoms, relationships, parenting, parental and sibling illness and stress, but it also reflects an appreciation of the challenges associated with an overfocus on the index or referred child (another notable barrier/imbalance to hearing the voice of the young person). Thus, CAMH services must balance parent/carer and Ch\&YP communications. ${ }^{1}$

The challenge, therefore, is to ensure that Ch\&YPs' voices are systematically 'heard' whilst acknowledging and incorporating parental (and others') concerns and difficulties in relationship work. One such approach is provided by The Family Model (TFM, Falkov, 2012), which helps clinicians to incorporate individual perspectives (both parent and Ch\&YP) into family focused care using a collaborative, non-judgemental process. This facilitates a balanced approach whereby Ch\&YPs' perspectives and those of key adults and other family members are considered when someone is unwell.

TFM provides clinicians with a structured clinical approach when engaging with the unwell person and their family members. The approach/method consists of a visual illustration of key areas/elements (Domains) and associated relational interactions (arrows), using a whiteboard or paper, to support a structured conversation between clinician and family members. The aim of this conversation is to acquire a shared understanding about how mental/physical illness in one family member can affect others and how they, through their understanding and responses can, in turn, influence the experience of the unwell person. TFM approach emphasises the core role of relationships in shaping experiences and determining outcomes for all family members. For example:

- Being a parent experiencing mental or physical illhealth can affect parenting, the interparental relationship and interactions with children;

- Ch\&YPs' mental and physical ill-health and developmental needs can impact on parents and other family members in various ways.

This article will focus on the latter example in $\mathrm{CAMH}$ services.

\section{Method}

Clinical vignettes will illustrate use of TFM in three different CAMH settings. The reporting of entire session ma- terial is beyond the scope of this article. Pseudonyms (as indicated by ${ }^{*}$ ) have been used and demographic aspects have been altered to preserve confidentiality. The vignettes will show how TFM helped clinicians to engage and work in partnership with all family members to achieve a shared understanding of the challenges they were facing. In doing so we hope to illustrate how TFM helps clinicians to achieve a balance between empowering Ch\&YPs' roles in managing their symptoms and developmentally appropriate input from their parents and other family members to enhance clinical outcomes. Stylised visual representations of de-identified TFM whiteboards have been provided to accompany the vignettes.

\subsection{The Family Model}

TFM visually demonstrates the key areas of focus and associated interactions using 6 Domains and 10 bidirectional arrows (Figure 1). The Domains include: Domain 1 (parental difficulties/illness), Domain 2 (Ch\&YP's illness/difficulties), Domain 3 (family relationships), Domain 4 (strengths and vulnerabilities), Domain 5 (services for Ch\&YP and for adults), and Domain 6 (a broader culture and community, ecological Domain).

TFM uses arrows to illustrate the bi-directionality and interdependence between Domains. The arrows represent the role of communication between all members when one or more individuals in a family are unwell. Falkov (as cited in Reupert, Maybery, \& Nicholson, 2015, pp. 8-9) notes that how the core components (Domains + arrows) interact with and influence each other is determined by the quality of an individual(s) adjustment within his/her family, as well as the adequacy of the whole family's adaptation to living with the individual(s) affected by illness.

\subsubsection{Using TFM Clinically}

TFM helps clinicians to visually identify and map ways in which family members are connected. The broad aim is to use TFM as a tool to foster engagement and to facilitate thinking about connections between symptoms and relationships in a balanced and understandable way, highlighting both the family's strengths and difficulties. Each person is provided with a photocopy of TFM (or TFM is drawn on a whiteboard). The approach is flexible and can be used with the unwell person (Ch\&YP or adult or both) and with other family members, in single or across multiple sessions.

This article examines the single session version. Here the focus is on broadening thinking about the unwell person's experience in the family and the impact of that person on others in the family and their associated responses. The aim is to facilitate a better shared understanding and to use this to improve communication about symptoms and their impacts by developing a collaborative, family focused care plan. In developing such

\footnotetext{
${ }^{1}$ Henceforth we use the term 'parent' for brevity, anticipating that our readers will consider this inclusive of caregivers and guardians.
} 


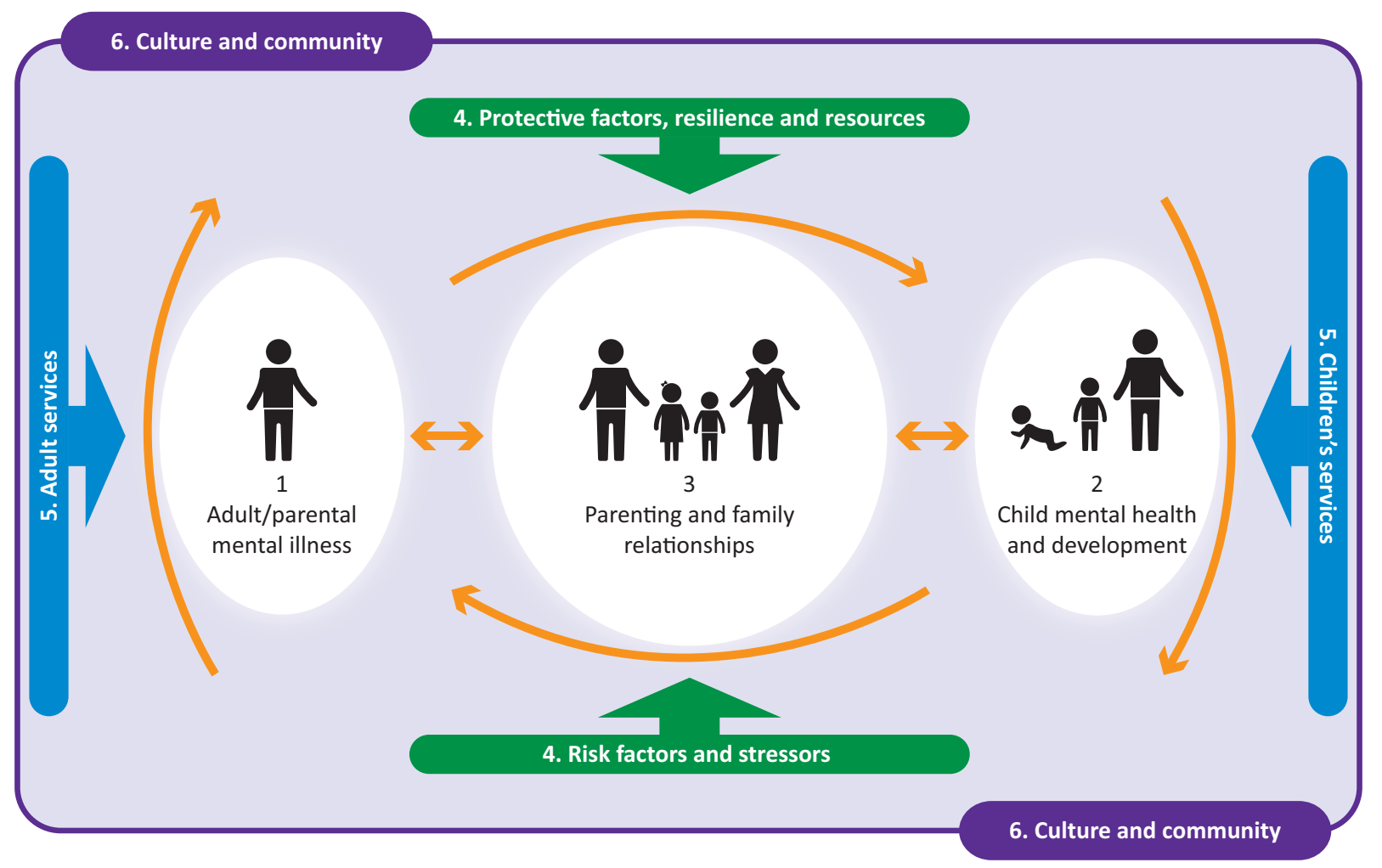

Figure 1. Cross-sectional components of TFM (Falkov, 2012).

a plan, the emphasis is on encouraging open discussion and empowering parents to engage with and respond to their Ch\&YP's concerns. This also helps to develop practical, age-appropriate strategies for change so that each person can respond in a way that is manageable for themselves and supportive of the unwell person. Specifically, TFM session concludes with those present actively contributing to a collaborative Care Plan.

\subsubsection{Overview of the Single Session Approach Using TFM}

The session begins with introductions and a brief explanation about the purpose of the meeting and use of TFM. This is followed by an explicitly agreed 'non-blaming, non-judgemental' approach. These words are written on the whiteboard or on paper, in red, to emphasise the core values associated with this approach.

The Six Domains then provide the foundation for a structured conversation, which proceeds sequentially, using the Domains and arrows. It starts by asking each person what they believe are the two key issues for them, at the time of the meeting. Their responses are recorded in summary form, according to respondent position (Adult-D1; Ch\&YP-D2).

In CAMH settings, the Ch\&YP is typically identified as the patient, and adult caregivers recognised as carers, but not necessarily as consumers with their own problems and predicaments. This can result in an over-focus on the identified patient's problems, as heard through the voices of carers. Where the Ch\&YP is the focus, TFM approach helps to illustrate the stress for adults and other family members when a Ch\&YP is unwell. It may also help to validate that others in the family may themselves also be struggling with significant stress or illness. This illustration of transgenerational difficulties helps to demonstrate the connectedness between, for example, the affected Ch\&YP and parents, and helps to reduce over-focus on just the unwell young person or child. This transgenerational connection between Domains 1 and 2 (adult and child mental health and development) is illustrated with arrows (which are drawn in red pen to emphasise the importance of the connections). This is a key point in the conversation where participants can observe, through their own responses, the connections between family members. This process is regarded as crucial in facilitating a shift in perspective, from an exclusive focus on the unwell individual to an appreciation of the interactions around that person.

Domain 3 (parenting and family relationships) is an opportunity to further acknowledge everyone's role in the family and the importance of communication. Examples are sought from family members to illustrate communication, including imbalances (recurring patterns associated with negative reactions, distress or frustration and anger). These imbalances, shared verbally by family participants, and recorded visually by clinicians on a whiteboard or paper, help to clarify problems and establish goals for the Care Plan. Clinicians emphasise that working together in this way can help recovery and focussing on communication can improve problem solving and risk management. 
An example of imbalanced communication is the tendency for parents accessing CAMH services to want their Ch/YP's problem 'fixed'. Whilst some parents can readily see that their role is important and that relationships are a key part of problem definition and resolution, in other instances there is a greater level of rigidity which might manifest as, for example, a reluctance to accept a role in their child's recovery. In such instances TFM has been helpful to improve parental appreciation of the relevance of family relationships as part of the Ch\&YP's treatment and recovery.

A further example of imbalance involves Ch\&YP who are in a caring role, for example in families where parental mental or physical illness is prominent, and where the extent of that role is proving to be sufficiently burdensome to impact on their development. By inviting each person to describe issues of importance to themselves in the presence of other family members, there is opportunity for improved understanding about each person's experience. This helps to illustrate both adult and child roles and highlights imbalances, in a non-blaming way. This can allow for more open conversation and mutual exploration of potential solutions via the care plan objectives.

Domain 4 (including risk and protective factors) validates the difficulties being faced, not only by the affected person but other family members too. This is essential for credible engagement in the next step, which focuses on strengths and protective factors. This balanced approach helps to empower family members in providing support and in developing recovery and relapse prevention strategies. The focus on strengths as well as difficulties helps to ensure that personal resources, which may have become hidden or neglected within the family's crisis, are acknowledged and incorporated into care planning and treatment approaches.

Domain 5 (services) provides an opportunity to reflect on the clinician's role and supports engagement. This Domain is based on the premise that prognosis is directly linked to the quality of the relationship between the family and key members of health-care teams. This is also the place to 'map' the services involved, their roles, and service gaps. A coordinated approach might include a multi-professional, inter-service meeting and consideration of the family's role. Confidentiality is of primary concern; clinician familiarity with good practice regarding information sharing, privacy and confidentiality helps ensure a balanced approach to any competing needs in the family. Respecting an individual's confidentiality is not in and of itself a barrier to thinking about and talking with family members.

Domain 6 (culture and community) allows for consideration of the family's experience of stigma and discrimination, neighbourhood and community support. This includes opportunity to consider cultural, religious, spiritual and migration issues which are often insufficiently addressed in $\mathrm{MH}$ services and which may be important accompaniments to the presenting symptoms and crucial determinants to recovery.

\subsubsection{Linkages}

The arrows connecting the six Domains provide clear visual evidence for participants to see how their respective experiences as individuals are linked. The sequential process of the collaborative conversation helps to build 'a visual picture of experiences' and assists each person with integrating the shared information. The aim is to develop a shared formulation of each family's unique 'story' in an empathic and empowering way, which respectfully reflects common themes such as loss, adversity and resilience. This enriched view reflects both individual and collective experience of symptoms and illness and helps to generate a developmentally informed, jointly owned Care Plan.

\subsection{Service Settings}

The service settings include an outpatient $\mathrm{CAMH}$ service, an inpatient specialist adolescent $\mathrm{MH}$ unit and a paediatric inpatient setting in North Sydney. Demographically this is an urban area with a population over 370,000 , significant cultural and linguistic diversity, with less than $60 \%$ Australian-born and $28 \%$ speaking a language other than English at home (Australian Bureau of Statistics, 2011). Mean income was higher than the national average and the unemployment rate lower.

\subsubsection{CAMH Community Outpatient Setting}

Northern Sydney Local Health District (NSLHD) Child and Youth MH Service (CYMHS) is a multidisciplinary specialist CAMH service located adjacent to a tertiary referral hospital. In 2016 a Quality Improvement (QI) initiative was undertaken to explore acceptability and feasibility of using TFM as a single session intervention. Referrals to the community outpatient clinic consisted of newly referred and existing clients and their families with two generations of mental ill-health, who were deemed to be 'complex' or 'stuck' by their referring clinician. TFM intervention was provided as a single, one-hour outpatient encounter with Ch\&YP and their family by members of the multidisciplinary CYMHS team. Clinicians also had a goal of enhancing collaborative care planning for the referred families. The $\mathrm{Q}$ I initiative collected written feedback from clients of the service, the content of which suggested TFM, as a single session, may be acceptable to Ch\&YP and their families (Hoadley, Falkov, \& Agalawatta, 2016).

\subsubsection{Specialist Adolescent Inpatient Setting}

The Brolga Unit is a purpose-built 12-bed adolescent $\mathrm{MH}$ acute inpatient unit within NSLHD, part of the CYMH service which accepts referrals from across New South Wales. It is expected that young people who require admission to the unit will have complex $\mathrm{MH}$ needs, often with other serious co-morbid psychosocial problems. The existing model of care entails work with the Ch\&YP 
and their parents to explore the impact and role of symptoms and problems across the family, complemented by evidence, values and strengths based approaches to treatment. An assessment of these systems forms an important part of case formulation and treatment planning. TFM was introduced in 2016 and was thought to be a potentially useful tool to enhance family focused practice, particularly to support formal recognition and communication of the bidirectional impact of family dynamics and the Ch\&YP's recovery.

The single session option has been used early in the admission (usually after initial individual sessions with the Ch\&YP and parents) and supports the expectation that assessment and treatment incorporates both the Ch\&YP and the family. The diagrammatic representation of the session is used to review progress and forms part of the discharge transition documentation.

\subsubsection{Paediatric Inpatient Setting}

The RNSH CYMHS service provides admissions for Ch\&YP to the RNSH paediatric ward. According to this ward's model of care, Ch\&YP who are at risk of significant harm to themselves, or who require containment to prevent deterioration can be admitted following the principles of least restrictive care. As part of the assessment and treatment planning for some of the young people on this ward, CYMHS clinicians provide a single session TFM intervention.

The purpose of TFM session within this setting is to gather information about the circumstances warranting admission and to assess and facilitate the changes that need to occur to allow safe discharge. The Ch\&YP's perspective is critical to this process as it is typically their communication concerning risk of harm to themselves that results in the need for admission. As admissions are intended to be brief and as the ward does not have the resources of a specialist $\mathrm{MH}$ unit, the focus of the session is to support the family in developing and agreeing on a care plan that supports discharge and transition to community-based services. The care plan may include, for example, the development of a system of communicating level of risk between parent and the Ch\&YP. The Ch\&YP is invited to discuss barriers to communication with parents, of particular importance where the Ch\&YP's comfort with communication may allow de-escalation, or even the prevention, of future crises. TFM allows for balanced consideration of differing perspectives concerning risk and emphasises roles and family strengths.

\section{Results}

\subsection{Vignettes}

\subsubsection{Community CAMH Outpatient Vignette}

Samuel* was a 14-year-old male who was referred to the outpatient $\mathrm{CAMH}$ service after inpatient care for a first episode of psychosis. Samuel initially presented to a tertiary hospital emergency department after several days of confusion and anxiety. The symptoms followed a 6-month period of social withdrawal and a decline in academic performance. There was no prior contact with mental health services and no history substance misuse. On Samuel's initial assessment behavioural disorganisation, auditory hallucinations and delusions of persecution, reference and control were described. There was a potential risk of harm to others consequent of thoughts of acting on the delusions of persecution involving his peer group. Samuel's inpatient management was predominantly biomedical and he achieved remission of positive symptoms and was discharged from inpatient care after a fortnight.

Samuel and his family's attendance at scheduled outpatient appointments was inconsistent. At appointments where he did attend, the community clinician was concerned by requests from the family to disengage from treatment. Attempts to involve the family in collaborative care planning resulted in apparent disengagement without evident alternative care plans. The clinician was concerned about Samuel's risk of relapse and about potential neglect of Samuel's medical care.

The complexity of the case and potential risk led to consultation with the local child protection service. With the assistance of the family's general practitioner, clinician concerns were communicated to the family who agreed to attend a care planning session as a family.

TFM was chosen to provide the framework for the session so as to explore family capacity to provide safe care and so that a shared understanding of Samuel's treatment needs could be established among clinicians and the family. Samuel's parents communicated up-front that, as they had received psycho-education, safety and relapse prevention plans as an inpatient and as Samuel was still taking his medication, they did not see a need for ongoing care. Samuel informed clinicians that though he had been adherent to date, he wanted to cease his medication. The clinician running the session was aware of the potential for conflict with the family and TFM provided the option to begin by exploring the family's strengths (Domain 4). Samuel volunteered that 'we box off the bad stuff' indicating that, from his perspective, the family managed potentially aversive experiences through avoidance. His parents endorsed this statement.

Subsequently, the clinician explored the impact of symptoms and illness across the two generations (Domains 1 and 2). Samuel spoke freely of his difficulty understanding why he had been admitted to hospital. This seemed to surprise his parents. His mother, in turn, spoke of a diagnosis of breast cancer one year earlier. She had received surgical and medical intervention for this with good response but had suffered from anxiety (Domain 1) in the context of this illness. Furthermore, when the clinician explored the impact of maternal medical illness on her mood (Domain 1), Samuel's mother stated, 'I never thought I was unwell', referring to the time after her cancer treatment. 
An exploration of the services involved in her medical care (Domain 5) indicated poor adherence to followup medical imaging and reluctance to attend her general practitioners' appointments due to time constraints. Samuel's mother had understandably avoided environments which might remind her of her own illness and the clinician perceived that Samuel, similarly, might be using this strategy to manage his own anxiety about illness and treatment. The clinician chose to highlight the parallels heard in the communications of mother and son, illustrating this with arrows connecting Domains 1 and 2 which allowed for more detailed discussion about communication as a family. The impact of the anxiety impacting on medical follow-up, as identified in both Domain 1 and 2, and how this might be addressed in a care plan was also demonstrated graphically (arrows from Domains 1 and 2 to care plan). The clinician noted that the family's strategy might be as much a challenge as it was at times a strength and reflected that it was Samuel's original statement about boxing off 'bad stuff' that proved the catalyst for family developing a shared understanding.

An exploration of Domain 6 allowed for a discussion of the family's migration story; Samuel and his parents were migrants from Columbia, having moved when he was 10 years of age. The dissimilarity of health services in Australia and the limitations to the availability of other family and community supports were noted by the clinician. This reflection served to emphasise both the role of the nuclear-family's strengths but also their need for health service support.

The care plan was constructed in collaboration with the family at the end of TFM session drawing on features from each of the other domains. As Samuel's primary concern had been duration of medication treatment, this included an explanation about the role of medication in relapse prevention. Samuel seemed to feel heard, as evidenced by his engagement in the conversation and participation in the development of the care plan. Samuel agreed to maintain adherence for an appropriate duration, pending review. Both Samuel and his mother made commitments to engage in outpatient management for their respective psychiatric and medical illnesses. Samuel and his parents requested for further family sessions to be part of Samuel's future care and contracted to several more sessions with the service.

Figure 2 demonstrates the reciprocal impact of Domain 1 and 2 concerns. Samuel and his mother worry about each other's wellbeing (depicted by the large red arrows and accompanying statements). The universal concern for the mother's illness and Samuel's primary concern (medication treatment) visually inform the care plan (green arrows).

THE FAMILY MODEL
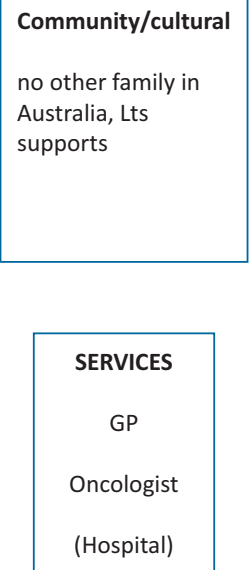

NONJUDGMENTAL
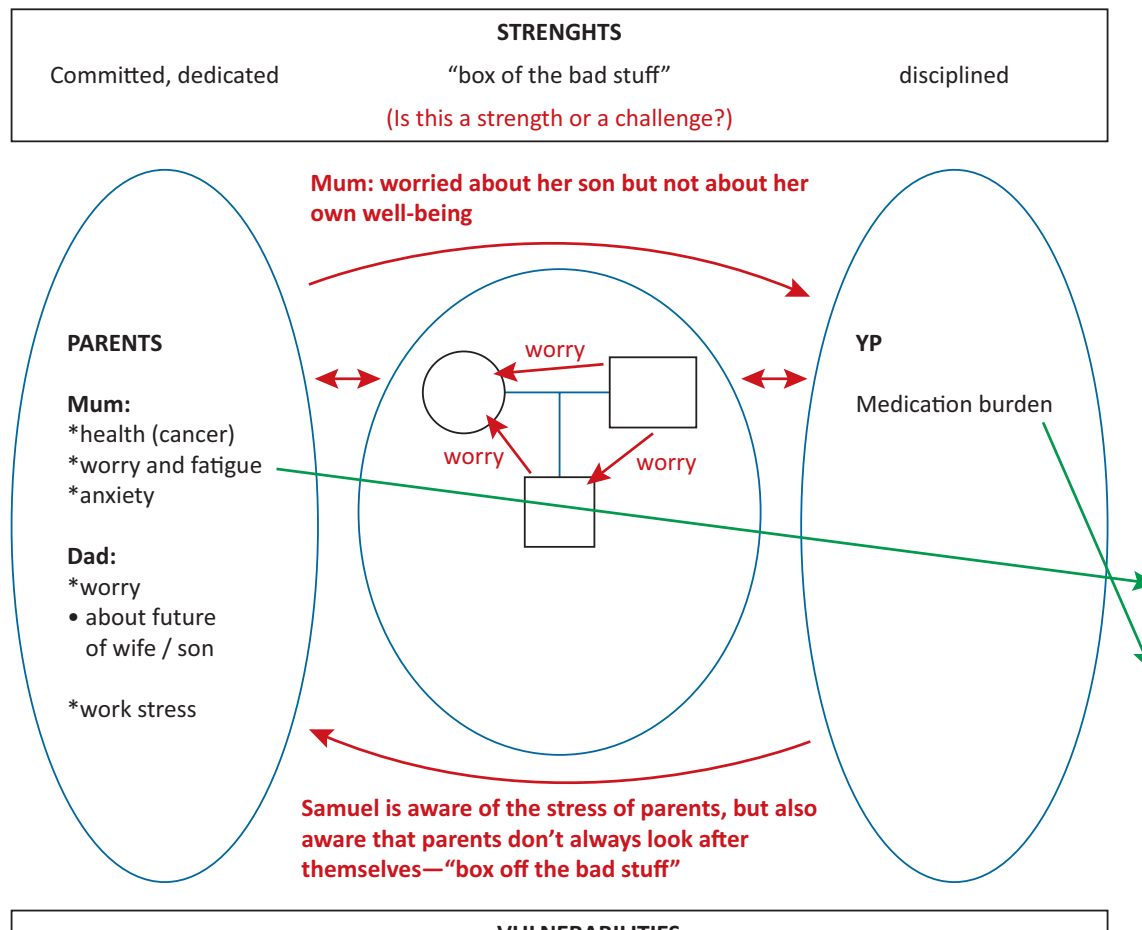

Mum: worried about her son but not about he own well-being

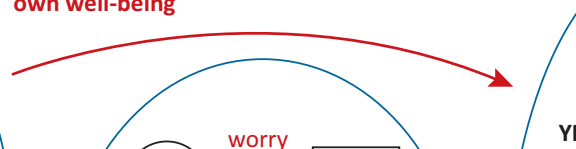

(physical, mental/stress)
NON-BLAME

SERVICES (YP)

- GP

(inconsistent)

- CYMHS (now)

- no school

counsellor?

CARE PLAN

To do:

check up with

- Medical Dr

- Psychiatry Dr

(medication

review)

Liaison with

school

counsellor

Crisis plan

To consider:

- family work

Figure 2. Outpatient TFM. 


\subsubsection{Specialist Adolescent Inpatient Vignette}

Jane* was a 15-year-old only child of Argentinian parents referred by the community CAMH service for 'diagnostic clarification'. Jane presented with symptoms of treatment resistant generalised anxiety disorder associated with deliberate self-harm and intrusive obsessional thoughts. She also reported recent onset perceptual disturbances in the context of worsening anxiety. She had not responded to high dose SSRI treatment and individual sessions with a psychologist. There was a history of prematurity and separation anxiety. Individual sessions indicated that both Jane and her parents act protectively towards each other as a priority. A single session TFM was used in the second week of her admission to facilitate discussion and develop a shared understanding of what the impact of individual anxieties and wellintended behaviours had on Janes' unremitting symptoms and their relationship.

During the TFM session, parents were asked about their two main issues. Both parents expressed their concern and care for Jane as their expected and understandable priority. Jane's mother acknowledged that her own anxiety (Domain 1) motivated her often intrusive interaction with Jane, particularly around health and nutritional issues. In contrast, Jane's father reflected how his anxiety about her wellbeing had led to his tendency to agree with Jane's requests. Jane identified worry and confusion as her main struggles (Domain 2). She was particularly worried about her parents' own worries and perceived her mother as intrusive as a result. This sharing of information, by each parent and young person, indicated important and difficult bidirectional connections across the generations.

This usefully illustrated the connections between Domains 1 and 2 and facilitated sequential progression of the conversation to Domain 3, where more detailed explorations of key communications occurred. For example, Jane described how her parents tended to catastrophize and panic in response to her distress. Jane also suggested that her mother's intrusiveness had impeded her ability to make her own choices about the management of distress. Both Jane and her parents also identified guilt as a barrier to talking about negative emotions and described consequent arguments and escalation of her anxiety symptoms.

As the conversation progressed Jane appeared able to speak more openly about how her mother's intrusiveness generated fear and withdrawal within herself. This was particularly empowering for Jane as her parents were helped to maintain the 'non-blame and non-judgemental' approach whilst she spoke. The clinician helped reframe Jane's mother's pattern of defensive-response as a need to provide for and protect Jane due to her fragility at birth. This allowed for discussion about adolescent developmental needs and age-appropriate parenting responses.

Domain 4 (strengths) were noted to be the family's capacity for connection through humour and their value on sharing time together, despite their difficulties. It was possible to validate these qualities as invaluable resources.

Domain 5 provided opportunity to review past service contacts and treatments and to highlight the gap in family focused work. This allowed for useful discussion about a balanced approach involving a combination of individual as well as family and relational components.

TFM provided a non-judgemental frame within which parents were able to better understand the origins of their anxieties (trauma of having a premature child without support), and how these well-intentioned expressions of anxiety impacted on Jane's mental health and development. In turn, Jane's avoidance of negative emotions led to behaviours that perpetuated her parents' worries and exacerbated her own anxieties. This vicious cycle of miscommunication was highlighted through the sequential process of TFM dialogue and illustrated using the bidirectional arrows (Figure 3).

A single TFM session helped to identify a pattern of transgenerational anxiety and to explore alternative strategies to communicate distress. The shared conversation also illustrated developmental perspectives in which all participants could appreciate their respective roles in both problematic communications and in potential solutions (Figure 3).

Jane's mood improved over a week-long admission and her perceptual disturbances dissipated without medication change. She was discharged home with ongoing outpatient follow-up, including a recommendation for ongoing family work to consolidate progress.

Figure 3 demonstrates the reciprocal impacts of Domain 1 and 2 concerns. In this case guilt concerning responsibility for a parent or young person's symptoms or vulnerability, respectively, are depicted by the large red arrows and accompanying statements. The absence of services for parents (Domain 5), despite the apparent vulnerabilities (Domain 4), is highlighted visually by red arrows and informs some of the items in the care plan.

\subsubsection{Paediatric Inpatient Vignette}

Abigail* was a 16-year-old Caucasian girl of Australian origin who lived with her parents and younger brother, Shaun*. She was referred to a tertiary hospital emergency department by her school counsellor with suicidal ideation and a suicide plan. She presented with symptoms of depressive disorder and of emotional dysregulation, with a history of both non-suicidal self-injury and of overdoses with suicidal intent. Although she had experienced some alleviation of symptoms during a prior 4month period of psychological treatment, her symptoms had escalated at a time when there were increasing academic demands and discord in the home environment.

Initial assessment indicated ongoing safety concerns and significant communication difficulties between Abigail and her parents. She was admitted to the paediatric ward with the aim of providing a brief ( $<72$ hour) inter- 
THE FAMILY MODEL

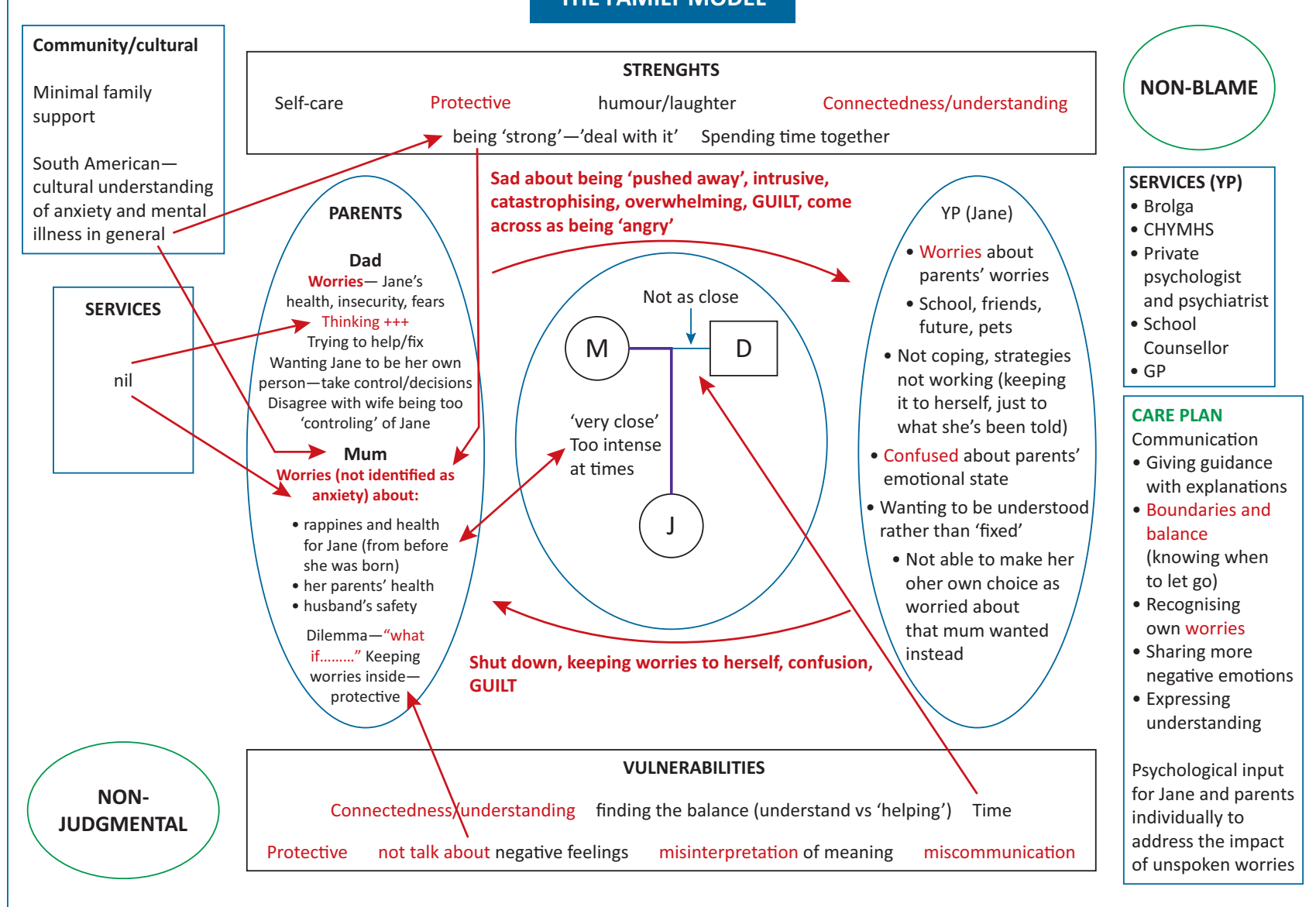

Figure 3. Specialist Adolescent Inpatient TFM.

vention, focused on safety and treatment planning. Following individual interviews with Abigail and her parents, a TFM session was arranged with the aim of developing a shared formulation of Abigail's crisis, the role and importance of communication, and a plan for discharge.

Abigail's parents noted difficulties balancing the demands of work with parenting and caring for the children's elderly grandmother. Both parents felt they had little time for themselves to exercise and relax (Domain 1). They also acknowledged high levels of interparental conflict (Domain 3), particularly with regards to the management of Shaun's externalising behaviours. There was anxiety (Domain 1) on her mother's behalf about the children's psychological health, and frustration on her father's part due to feeling ineffective and helpless in supporting and managing the family as he felt he should.

With her parents' concerns acknowledged, Abigail could discuss her increasingly low mood and anxiety (Domain 2), and explore her reluctance to communicate this to her parents. Abigail pointed out that she perceived her parents as overloaded and this was supported by the information visually depicted in the TFM model (Domain 1). Over time, Abigail's withdrawal had reduced opportunities for developing a close, trusting relationship with her parents and she did not see them as capable of assisting her to manage her distress (Domain 3). Furthermore, she feared that additional stress might precipitate their separation. This communication prompted her parents to reassure her that there was no intention for the parental unit to break apart.

Thick red arrows were drawn between Domains 1 and 2, illustrating the importance of the connection between Ch\&YP distress and the overloaded parental unit. Of particular importance was the clinician's ability to link some of this information to Domain 4. The strengths included Abigail's parents expressing their commitment to her support. Additionally, her parents could empathise and validate how hard this experience must be for her given her concern that they were overloaded and might separate.

All agreed that being closely connected as a family was both a strength and a weakness; the family reported sensing, but also fuelling, each other's stress. This provided opportunity to explore family interactions in more detail in Domain 3. Abigail explained that she had invested additional effort in academic achievement in an attempt to compensate for parental stress and she indicated disappointment that this had not helped the situation at home (Domain 3). Abigail's mother acknowledged that she too had immersed herself in her own work as a refuge from worry about her children (Domain 1 and 3). 
The clinician discussed this interdependence between the children's and parents' difficulties (Domain 3) and used arrows to depict this relationship.

The clinician helped Abigail's mother to recognise that to avoid her own distress she may not have attended to signs of Abigail's anxiety. This avoidance was reframed as self-protective and as a behaviour that allowed her to function as a provider. This avoidant coping style had also frustrated Abigail's father and created tension between them (Domain 3), specifically as it had not been effective in managing Shaun's behaviours. The clinician suggested that though Abigail's mother's focus on employment was accepted as a strength, the accompanying avoidance at home was seen as a vulnerability (Domain 4). Abigail's father explained that he felt his son's behaviour was the cause of many arguments in the home. Reflecting the over-focus of his father, Shaun contributed that he often sensed that he was blamed and felt in the spotlight for the wrong reasons (Domain 3). Shaun reported resentment toward his parents, and his parents reflected that this might have increased his provocative behaviours and distanced him from the family, whilst also reducing their capacity to respond to Abigail's needs. Again, the interdependence between Domains 1,2 and 3 was visually represented with arrows to aid the family's understanding.
In devising the Care Plan to support Abigail's safe discharge, a system to communicate perceived risk that placed equal responsibility on Abigail and her parents was discussed. This was intended to address some of the challenges identified in Domain 3 and 4. As is typical, the communication system intended to allow Abigail to disclose risk in a clear and simple manner. Pre-agreed responses were aimed at minimising potential escalation of risk, and over time to support development of trust in consistent parental responses (Domain 3).

Although not discussed in the single TFM session above, the impact of Domain 6 was implicit in the conversation about academic and occupational behaviours. The pressures experienced by a family living in a developed urban area, in which it is commonplace for both parents to work full-time and where the expectations of adolescents to compete with peers to achieve at school formed part of the cultural pressures impacting on this family.

Figure 4 demonstrates the impact of the Domain 1 concerns on the young person's symptoms (the large red arrow and accompanying statement). Much of the material was understandably challenging for the family and strengths in Domain 4 are emphasised visually. Each Ch\&YP's communication about their $\mathrm{MH}$ concerns and challenges (in addition to parental input) informs the care plan (smaller red arrows).

\section{THE FAMILY MODEL}

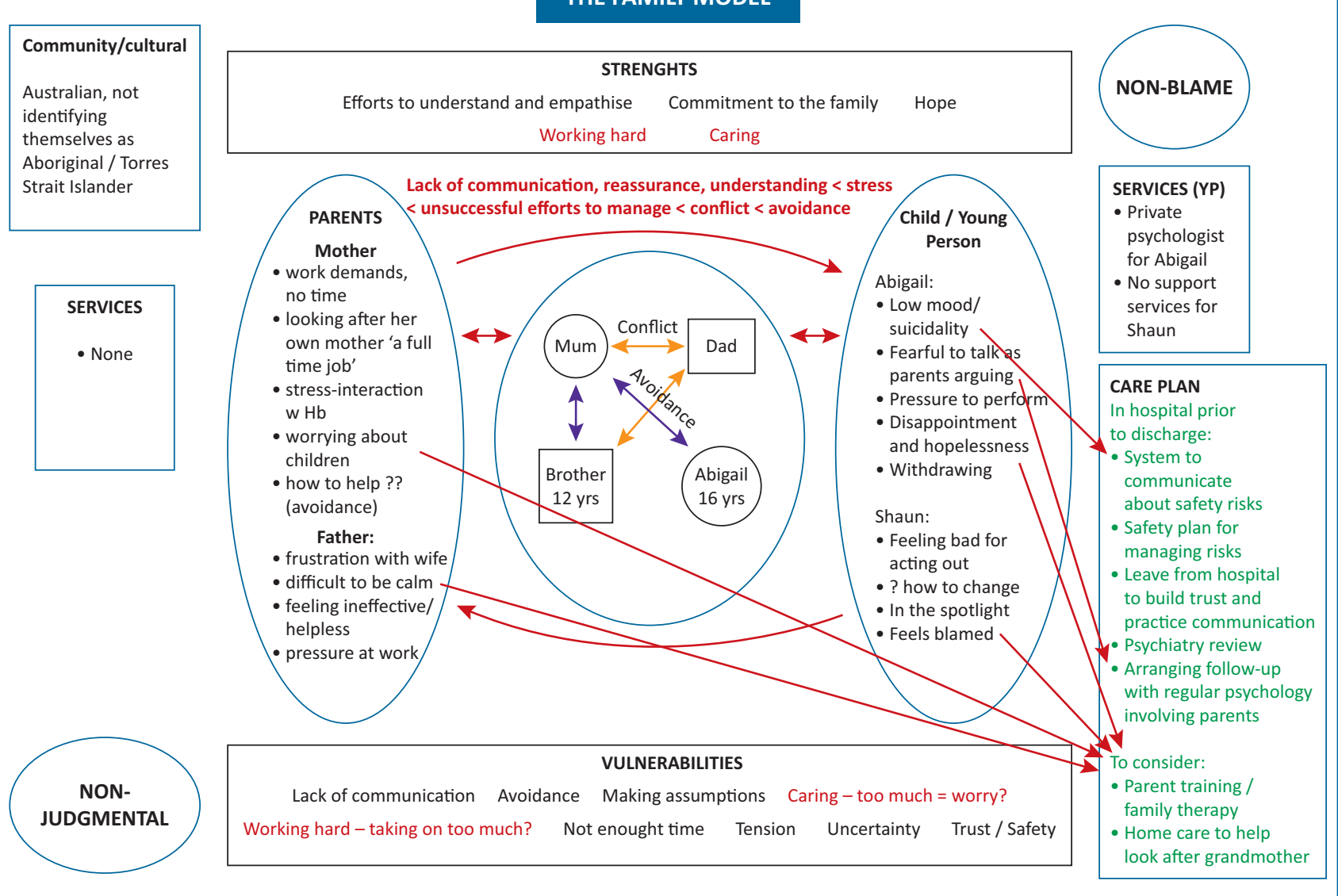

Figure 4. Paediatric inpatient TFM. 


\section{Discussion}

These vignettes help to illustrate the potential usefulness of TFM as a feasible, accessible and understandable intervention for both clinicians and family members in a range of CAMHS settings. The approach appears to support clinician engagement with both parents and their unwell Ch\&YP in achieving improved communication and collaborative care planning. Despite clinical complexity/acuity, a balance was achieved between enabling the voice of the young person to be heard whilst ensuring understandable parental concerns about their child were appropriately attended to.

Despite the varied clinical settings and the diversity of patient predicament, clinicians were able to use TFM approach to ensure the 'voice' of the YP was heard in a way that was meaningful to parents, and so that the parents in turn were helped to better appreciate their own roles as both part of the clinical complexity and integral to the care planning process. Clinicians found that TFM frame together with the sequential conversation process and the communications of the young person (the identified patient in CAMH services) seemed to assist parental acknowledgment of their own challenges. For example, in Vignette 1 Samuel's communication indicated his frustration with the CAMH inpatient service and his mother subsequently provided an important insight into her own pattern of accessing care. In Vignette 2 Jane reported chronic withdrawal to minimise parental anxiety, paving the way for her mother to speak of her own fears for her daughter's vulnerability. In Vignette 3, Abigail's voice highlighted parental discord allowing her parents to speak about their own relationship, a taboo topic for the family previously.

The young person's voice also consistently allowed for collaborative care planning. Samuel expressed fears about lengthy medication prescription and the therapeutic alliance was improved by focusing on this in the development of the care plan. Jane's communication indicated repression of negative affect to protect herself from parental response and the care plan highlighted the role of parental anxiety and its consequences for the community service providing follow-up. Finally, Abigail spoke of a withdrawal from communication at home to protect what she saw as a fragile parental unit. As a result, parent-child communication strategies in the home environment were prioritised to assist with altering communication patterns to support safe discharge planning.

TFM was implemented at similar times across the three locations and though the above commonalities emerged, the implementation at sites with different practices and clinical priorities resulted in some unique elements. In the outpatient service, the use of TFM focused on families known to already be burdened by two generations of mental illness, and the impact of symptoms between parent illness (Domain 1) and the development of child illness (Domain 2) was central to the use of TFM. In the specialist adolescent unit, the use of TFM evolved to support systematic family focused practice, and use as a discharge transition tool-communication to the outpatient service at time of discharge. The inpatient service could highlight visually what areas (Domains) were priorities for ongoing work, enhancing communication with the Ch\&YP's community clinician and emphasising the importance on ongoing FFP.

TFM allowed for a more realistic and transparent formulation to be communicated not only during the Ch\&YP's admission, but it also formed a thread through which ongoing treatment could be based. In the inpatient context, TFM facilitated discharge planning by enabling the Ch\&YP to give their perspective about their strengths, in addition to stressors, triggers and relationships that may have contributed to a crisis. In this way, the Ch\&YPs' communications assisted with the management of risk and in helping clinicians and the family consider options for the near future.

\section{Limitations}

This is a small series of single case vignettes which presents anecdotal evidence suggesting the utility of TFM as a tool for brief family focused work in different service settings. Though the approach has potential as an accessible way of promoting family focussed work and improving collaborative practice there is a need for further evaluation, applying rigorous trial methodology and using larger samples to establish efficacy and effectiveness.

We reported improvement in the clinical presentations of the young people described in the vignettes. We encourage caution in the assumption that such improvement is maintained in the longer term. Single session psychotherapy has been described as efficacious and costeffective (Talmon, 2012, pp. 7-8) and single session family therapy has recently been reported to be an effective tool in improving well-being for young people in the Australian context (Hopkins, Lee, McGrane, \& Barbara-May, 2017 , p. 110). Whether the collaborative care planning impacted on the long-term outcomes for the Ch\&YP and their families is not within the scope of this article but is an important issue in need of further research to establish the efficacy and effectiveness of TFM as a single session intervention. Future work will also need to evaluate instances where the model does not demonstrate benefit and ultimately will need to look at overall efficacy data and cost indices of implementing TFM.

\section{Conclusions}

These vignettes suggest that TFM use as a single session tool provides an opportunity for clinician-mediated communication between the Ch\&YP and parents, whereby the Ch\&YP can voice concerns in a non-judgemental, non-blaming frame and where clinicians might model adaptive communication styles and strategies. The Ch\&YPs' voices were not heard in isolation and were always interconnected with those of their families. It is 
notable that TFM acted as a conduit for clinicians with varied clinical backgrounds, across different sites whilst meeting diverse patient predicaments to facilitate empathetic communication within the family and the empowerment of the Ch\&YP.

This experience to date suggests the approach worthy of more systematic evaluation to test the potential efficacy of TFM, with clinician skills acquisition (provision of training), family experience of the approach, and clinical outcome assessment.

\section{Acknowledgements}

The authors would like to acknowledge the participation and feedback of the families, and the clinical and governance contributions of the staff of the NSLHD CYMHS services and of the RNSH children's ward.

\section{Conflict of Interests}

No conflict of interest.

\section{References}

Australian Bureau of Statistics. (2011). Statistical Area Level 4, Sydney-North Sydney and Hornsby, 2011 Census Quickstats. Retrieved from http://www.censusdata.abs.gov.au/census_services/ getproduct/census/2011/quickstat/121?opendocum ent\&navpos=95

Australian Infant, Child, Adolescent and Family Mental Health Association. (2008). National youth participation strategy (NYPS) in mental health: Strategy scoping project report. Stepney, Australia: AICAFMHA.

Children and Young Persons (Care and Protection) Act (1998) (NSW) No 157 (Austl.). Retrieved from http://www.legislation.nsw.gov.au/\#/view/act/1998 $/ 157$

Convention on the Rights of the Child. (1989). Retrieved from https://treaties.un.org/doc/Publication/MTDS G/Volume I/Chapter IV/iv-11.en.pdf

Day, C. (2008). Children's and young people's involvement and participation in mental health care. Child and Adolescent Mental Health, 13(1), 2-8. http:// doi.org/10.1111/j.1475-3588.2007.00462.x

Falkov, A. (2012). The Family Model handbook. An integrated approach to supporting mentally ill parents and their children. East Sussex: Pavilion Publishing.

Foster, K., Maybery, D., Reupert, A., Gladstone, B., Grant, A., Ruud, T., . . . Kowalenko, N. (2016). Familyfocused practice in mental health care: An integrative review. Child \& Youth Services, 37(2), 129-155. http://doi.org/10.1080/0145935X.2016.1104048

Hoadley, B., Falkov, A., \& Agalawatta, N. (2016). The complex case clinic: A family-focused approach to two-generation mental illness in families known to child and youth mental health services. Australian \& New Zealand Journal of Psychiatry, 50(S1), 3-170. http://doi.org/10.1177/0004867416640967

Hopkins, L., Lee, S., McGrane, T., \& Barbara-May, R. (2017). Single session family therapy in youth mental health: Can it help? Australasian Psychiatry, 25(2), 108-111. http://doi.org/10.1177/103985 6216658807

Macdonald, E., Lee, E., Geraghty, K., McCann, K., Mohay, H., \& O’Brien, T. (2007). Towards a developmental framework of consumer and carer participation in child and adolescent mental health services. Australasian Psychiatry, 15(6), 504-508. http://doi.org/ $10.1080 / 10398560701458228$

Reupert, A., Maybery, D., \& Nicholson, J. (2015). Towards the development of a conceptual framework. In A. Reupert, D. Mayberry, J. Nicholson, M. Gopfert, \& M. Seeman (Eds.), Parental psychiatric disorder: Distressed parents and their families, (3rd ed.). Cambridge: Cambridge University Press. http:// doi.org/10.1017/CBO9781107707559

Rutter, M., \& Stevenson, J. (2010). Developments in child and adolescent psychiatry over the last 50 years. In S. M. J. Rutter, D. Bishop, D. Pine, S. Scott, J. S. Stevenson, E. A. Taylor, \& A. Thapar (Eds.), Rutter's child and adolescent psychiatry. (5th ed.). Massachusetts: Wiley-Blackwell. http://doi.org/http:// dx.doi.org/10.1002/9781444300895

Talmon, M. (2012). When less is more: Lessons from 25 years of attempting to maximize the effect of each (and often only) therapeutic encounter. Australian and New Zealand Journal of Family Therapy, 33(1), 6-14. http://doi.org/Doi 10.1017/Aft.2012.2

Weil, L. G., Lemer, C., Webb, E., \& Hargreaves, D. S. (2015). The voices of children and young people in health: where are we now? Archives of Disease in Childhood, 100(10), 915-917. http://doi.org/ 10.1136/archdischild-2014-307492

\section{About the Authors}

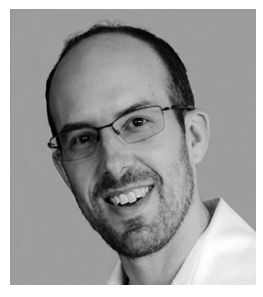

Benjamin Hoadley is a Child Psychiatrist in Sydney. He completed undergraduate medicine in New Zealand and subsequently specialist psychiatric training in Australia. Dr. Hoadley works within public outpatient and inpatient child, adolescent \& youth mental health services in addition to working with an early intervention program for conduct-related problems in children. His interests include familyfocused work and mental health education. 


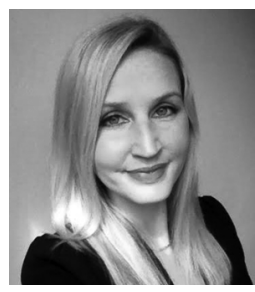

Freya Smith is a Clinical Psychologist who works with children and adolescents in an acute hospital setting and with adults in private practice. She completed a 3-year Bachelor of Science with Honours at the University of York (England), followed by a 1-year Post Graduate Diploma in Psychology and a 3-year Doctorate in Clinical Psychology at Massey University (New Zealand). Dr. Smith has research experience and ongoing interest in emotion regulation and psychological wellbeing related to physical health.

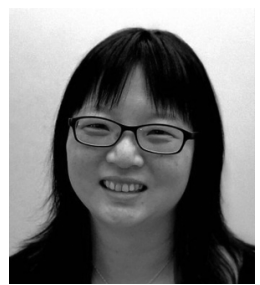

Cecilia Wan is a specialist Child, Adolescent and Family Psychiatrist. She completed her medical degree at the University of Sydney. Dr. Wan achieved additional specialist qualification as a Child and Adolescent Psychiatrist and has extensive experience helping new parents, children, adolescents and families understand, manage and minimise the impact of mood and behavioural disorders. She has worked extensively in both community and in-patient settings throughout Sydney.

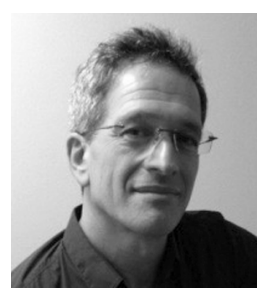

Adrian Falkov is a consultant child, adolescent and family psychiatrist in Sydney, Australia. He has longstanding interests in the links between adult and child experience of mental illness and the interplay between psychiatric symptoms and family relationships. These interests have informed family focused work in mental health services using The Family Model (www.thefamilymodel.com). This integrated partnership approach, based on shared expertise (professional and 'lived experience'), supports collaboration between clinicians, carers, consumers and their families. 